\title{
Necessary and sufficient conditions for generalized convexity
}

\author{
by JANUsz KRZYSZKOWSKI (Kraków)
}

\begin{abstract}
We give some necessary and sufficient conditions for an $n-1$ times differentiable function to be a generalized convex function with respect to an unrestricted $n$ parameter family.
\end{abstract}

1. Introduction. A family $F$ of continuous real-valued functions $\varphi$ defined on an open interval $(a, b)$ is said to be an $n$-parameter family on $(a, b)$ (see [1] and [5]) if for any distinct points $x_{1}, \ldots, x_{n}$ in $(a, b)$ and any numbers $y_{1}, \ldots, y_{n}$ there exists exactly one $\varphi \in F$ satisfying

$$
\varphi\left(x_{i}\right)=y_{i}, \quad i=1, \ldots, n .
$$

Throughout the paper we assume $n \geq 2$.

Let $F$ be an $n$-parameter family on $(a, b)$. Following [5] we say that a function $\psi$ continuous on $(a, b)$ is strictly $F$-convex $(F$-convex, strictly $F$-concave, $F$-concave $)$ on $(a, b)$ if for any points $a<x_{1}<\ldots<x_{n}<b$ the unique $\varphi \in F$ determined by

$$
\varphi\left(x_{i}\right)=\psi\left(x_{i}\right), \quad i=1, \ldots, n,
$$

satisfies the inequalities

$$
(-1)^{n+i} \varphi(x)<(\leq,>, \geq)(-1)^{n+i} \psi(x), \quad x \in\left(x_{i}, x_{i+1}\right),
$$

for $i=0,1, \ldots, n$, where $x_{0}:=a$ and $x_{n+1}:=b$.

The above inequalities can be rewritten as

$$
\operatorname{sgn}(\psi(x)-\varphi(x))=\operatorname{sgn}\left(\prod_{i=1}^{n}\left(x-x_{i}\right)\right), \quad x \in(a, b),
$$

1991 Mathematics Subject Classification: Primary 26A51.

Key words and phrases: generalized convexity, unrestricted $n$-parameter family. 
for strict convexity and

$$
\operatorname{sgn}(\psi(x)-\varphi(x))=-\operatorname{sgn}\left(\prod_{i=1}^{n}\left(x-x_{i}\right)\right), \quad x \in(a, b),
$$

for strict concavity.

A family $F$ of $C^{n-1}$ functions on $(a, b)$ is called an unrestricted n-parameter family (or briefly an $H_{n}$-family) on $(a, b)$ (see [3]) if for any distinct $x_{1}, \ldots, x_{k} \in(a, b)$, any positive integers $\lambda_{1}, \ldots, \lambda_{k}$ such that $\lambda_{1}+\ldots+\lambda_{k}=n$, and any numbers $y_{i}^{\mu_{i}}$, where $i=1, \ldots, k, \mu_{i}=0, \ldots, \lambda_{i}-1$, there exists exactly one $\varphi \in F$ satisfying

$$
\varphi^{\mu_{i}}\left(x_{i}\right)=y_{i}^{\mu_{i}}, \quad i=1, \ldots, k, \mu_{i}=0, \ldots, \lambda_{i}-1,
$$

where

$$
\varphi^{0}(x):=\varphi(x), \quad \varphi^{l}(x): \frac{d^{l} \varphi(x)}{d x^{l}} \quad \text { for } l=1,2, \ldots
$$

This notation will be used throughout the paper.

It is evident that any $H_{n}$-family on $(a, b)$ is an $n$-parameter family on $(a, b)$. Therefore we may consider the generalized convexity with respect to $H_{n}$-families. To begin with we introduce the following definitions:

Let $F$ be an $H_{n}$-family on $(a, b)$ and let $\psi$ be $n-1$ times differentiable on $(a, b)$. Let $i_{1}, \ldots, i_{k}$ be positive integers such that $i_{1}+\ldots+i_{k}=n$. The function $\psi$ will be said to satisfy the condition $W_{n}\left(i_{1}, \ldots, i_{k} ; F\right)$ (resp. $\widetilde{W}_{n}\left(i_{1}, \ldots\right.$ $\left.\left.\ldots, i_{k} ; F\right)\right)$ on $(a, b)$ if for any $a<x_{1}<\ldots<x_{k}<b$,

$$
\operatorname{sgn}(\psi(x)-\varphi(x))=\operatorname{sgn}\left(\prod_{l=1}^{k}\left(x-x_{i}\right)^{i_{l}}\right), \quad x \in(a, b)\left(\text { resp. } x \in\left(x_{1}, x_{k}\right)\right),
$$

where $\varphi \in F$ is determined by

$$
\varphi^{j_{l}}\left(x_{l}\right)=\psi^{j_{l}}\left(x_{l}\right), \quad l=1, \ldots, k, j_{l}=0, \ldots, i_{l}-1 .
$$

The function $\psi$ will be said to satisfy the condition $K_{n}\left(i_{1}, \ldots, i_{k} ; F\right)$ (resp. $\left.\widetilde{K}_{n}\left(i_{1}, \ldots, i_{k} ; F\right)\right)$ on $(a, b)$ if for any $a<x_{1}<\ldots<x_{k}<b$,

$\operatorname{sgn}(\psi(x)-\varphi(x))=-\operatorname{sgn}\left(\prod_{l=1}^{k}\left(x-x_{i}\right)^{i_{l}}\right), \quad x \in(a, b)\left(\right.$ resp. $\left.x \in\left(x_{1}, x_{k}\right)\right)$, where $\varphi \in F$ is determined by (4).

We will use the symbol $\varphi\left(x_{1}^{i_{1}}, \ldots, x_{k}^{i_{k}} ; \psi ; \cdot\right)$ to denote the function $\varphi \in F$ satisfying (4).

It is well known (see [3]) that $\psi$ is strictly $F$-convex on $(a, b)$ iff for any $a<x_{1}<\ldots<x_{n}<b$ the function $\varphi$ determined by (1) satisfies (2) on $\left(x_{1}, x_{n}\right)$. This means that $\psi$ satisfies the condition $W_{n}\left(1^{(n)} ; F\right)$ on $(a, b)$ iff $\psi$ satisfies the condition $\widetilde{W}_{n}\left(1^{(n)} ; F\right)$ on $(a, b)$. Here $1^{(n)}$ stands for $\underbrace{1, \ldots, 1}_{n}$. 
It is of interest to know whether the conditions $W_{n}\left(i_{1}, \ldots, i_{k} ; F\right)$ are equivalent to strict $F$-convexity.

The case $n=2$ was considered by D. Brydak [2]. He has proved that if $F$ is an $H_{2}$-family on $(a, b)$ and $\psi$ is differentiable on $(a, b)$, then $\psi$ is strictly $F$-convex on $(a, b)$ iff $\psi$ satisfies $W_{2}(2 ; F)$ on $(a, b)$.

The case $n=3$ was considered by the author in [4]. The theorem in [4] reads as follows: Let $F$ be an $H_{3}$-family on $(a, b)$ and let $\psi$ be twice differentiable on $(a, b)$. Then the conditions

- $\psi$ is strictly $F$-convex on $(a, b)$;

- $\psi$ satisfies $W_{3}(1,2 ; F)$ on $(a, b)$;

- $\psi$ satisfies $W_{3}(3 ; F)$ on $(a, b)$;

- $\psi$ satisfies $W_{3}(2,1 ; F)$ on $(a, b)$

are equivalent.

We will prove the following two theorems: Let $F$ be an $H_{n}$-family on $(a, b)$ and let $\psi$ be $n-1$ times differentiable on $(a, b)$.

1. If $\psi$ is strictly $F$-convex on $(a, b)$, then for any positive integers $i_{1}, \ldots, i_{k}$ such that $i_{1}+\ldots+i_{k}=n, \psi$ satisfies $W_{n}\left(i_{1}, \ldots, i_{k} ; F\right)$ on $(a, b)$.

2. If $\psi$ satisfies $W_{n}\left(i_{1}, \ldots, i_{k} ; F\right)$ on $(a, b)$ for some $i_{1}, \ldots, i_{k} \in\{1,2,3\}$ such that $i_{1}+\ldots+i_{k}=n$, then $\psi$ is strictly $F$-convex on $(a, b)$.

\section{Lemmas}

Lemma 1. Let $f$ and $g$ be defined and $k$ times differentiable in a neighbourhood of a point $x_{0}$ and let

$$
f^{i}\left(x_{0}\right)=g^{i}\left(x_{0}\right), \quad i=0,1, \ldots, k-1 .
$$

(i) If there exists a sequence $\left\{x_{n}\right\}$ such that $x_{n} \rightarrow x_{0}^{+}$and $f\left(x_{n}\right) \geq$ $g\left(x_{n}\right)$ for $n=1,2, \ldots$, then $f^{k}\left(x_{0}\right) \geq g^{k}\left(x_{0}\right)$.

(ii) If there exists a sequence $\left\{x_{n}\right\}$ such that $x_{n} \rightarrow x_{0}^{-}$and $f\left(x_{n}\right) \geq$ $g\left(x_{n}\right)$ for $n=1,2, \ldots$, then $(-1)^{k} f^{k}\left(x_{0}\right) \geq(-1)^{k} g^{k}\left(x_{0}\right)$.

(iii) If there exists a sequence $\left\{x_{n}\right\}$ such that $x_{n} \rightarrow x_{0}^{+}\left(\right.$or $\left.x_{n} \rightarrow x_{0}^{-}\right)$ and $f\left(x_{n}\right) \geq g\left(x_{n}\right) \geq 0$ for $n=1,2, \ldots$, and $f^{i}\left(x_{0}\right)=0$ for $i=0,1, \ldots, k$, then $g^{k}\left(x_{0}\right)=0$.

We omit an easy proof.

The two lemmas below are easy consequences of the definitions of $W_{n}\left(i_{1}, \ldots, i_{k} ; F\right)$ and $K_{n}\left(i_{1}, \ldots, i_{k} ; F\right)$.

Lemma 2. Let $i_{1}, \ldots, i_{k}$ be positive integers such that $i_{1}+\ldots+i_{k}=n$. Then the following conditions are equivalent:

- If $G_{1}$ is an $H_{n}$-family on $(a, b), \psi_{1}$ is $n-1$ times differentiable on $(a, b)$ and $\psi_{1}$ is strictly $G_{1}$-convex on $(a, b)$, then $\psi_{1}$ satisfies $W_{n}\left(i_{1}, \ldots, i_{k} ; G_{1}\right)$ on $(a, b)$. 
- If $G_{2}$ is an $H_{n}$-family on $(a, b), \psi_{2}$ is $n-1$ times differentiable on $(a, b)$ and $\psi_{2}$ is strictly $G_{2}$-concave on $(a, b)$, then $\psi_{2}$ satisfies $K_{n}\left(i_{1}, \ldots, i_{k} ; G_{2}\right)$ on $(a, b)$.

LEMMA 3. Under the assumptions of Lemma 2 the following conditions are equivalent:

- If $G_{1}$ is an $H_{n}$-family on $(a, b), \psi_{1}$ is $n-1$ times differentiable on $(a, b)$ and $\psi_{1}$ satisfies $W_{n}\left(i_{1}, \ldots, i_{k} ; G_{1}\right)$ on $(a, b)$, then $\psi_{1}$ is strictly $G_{1}$-convex on $(a, b)$.

- If $G_{2}$ is an $H_{n}$-family on $(a, b), \psi_{2}$ is $n-1$ times differentiable on $(a, b)$ and $\psi_{2}$ satisfies $K_{n}\left(i_{1}, \ldots, i_{k} ; G_{2}\right)$ on $(a, b)$, then $\psi_{2}$ is strictly $G_{2}$-concave on $(a, b)$.

The proofs of the next lemmas are not so simple.

Lemma 4. Let $F$ be an $H_{n}$-family on $(a, b)$ and let $\psi$ be $n-1$ times differentiable on $(a, b)$. If $\psi$ satisfies $\widetilde{W}_{n}(n-1,1 ; F)$ and $\widetilde{W}_{n}(1, n-1 ; F)$ on $(a, b)$, then $\psi$ satisfies $W_{n}(n ; F)$ on $(a, b)$.

Proof. We have to show that for any $x_{0} \in(a, b)$,

$$
\operatorname{sgn}\left(\psi(x)-\varphi_{1}(x)\right)=\operatorname{sgn}\left(\left(x-x_{0}\right)^{n}\right), \quad x \in(a, b),
$$

where $\varphi_{1}(x):=\varphi\left(x_{0}^{n} ; \psi ; x\right), x \in(a, b)$. We prove this equality on $\left(x_{0}, b\right)$; the proof for $\left(a, x_{0}\right)$ is analogous.

It suffices to show that

$$
\psi(x)>\varphi_{1}(x), \quad x \in\left(x_{0}, b\right) .
$$

Assume that this inequality does not hold. Then two cases are possible:

1. $\psi(x) \geq \varphi_{1}(x)$ for $x \in\left(x_{0}, b\right)$ and $\psi(c)=\varphi_{1}(c)$ for a $c \in\left(x_{0}, b\right)$

2. $\psi(c)<\varphi_{1}(c)$ for a $c \in\left(x_{0}, b\right)$.

1. It is easily seen that $\varphi\left(x_{0}^{n-1}, c^{1} ; \psi ; x\right)=\varphi_{1}(x)$ for $x \in(a, b)$. Since $\psi$ satisfies $\widetilde{W}_{n}(n-1,1 ; F)$ on $(a, b)$, this gives $\psi(x)<\varphi_{1}(x)$ for $x \in\left(x_{0}, c\right)$, which contradicts 1 .

2. Set

$$
\varphi_{2}(x):=\varphi\left(x_{0}^{n-1}, c^{1} ; \psi ; x\right), \quad x \in(a, b) .
$$

Since $\psi$ satisfies $\widetilde{W}_{n}(n-1,1 ; F)$ on $(a, b)$, we have

$$
\psi(x)<\varphi_{2}(x), \quad x \in\left(x_{0}, c\right) .
$$

It follows from the definitions of $\varphi_{1}, \varphi_{2}$ and from $\psi(c)<\varphi_{1}(c)$ that

$$
\begin{aligned}
\varphi_{1}^{i}\left(x_{0}\right) & =\varphi_{2}^{i}\left(x_{0}\right), \quad i=0,1, \ldots, n-2, \\
\varphi_{1}(c) & >\varphi_{2}(c) .
\end{aligned}
$$

We conclude from $(6)$ and $(7)$ that $\varphi_{1}(x) \neq \varphi_{2}(x)$ for $x \neq x_{0}$, because $\varphi_{1}, \varphi_{2} \in F$ and $F$ is an $H_{n}$-family on $(a, b)$, whence $\varphi_{1}(x)>\varphi_{2}(x)$ for 
$x \in\left(x_{0}, b\right)$, and finally, $\psi(x)<\varphi_{2}(x)<\varphi_{1}(x)$ for $x \in\left(x_{0}, c\right)$, by $(5)$. We can rewrite the last inequalities as follows:

$$
0<\varphi_{1}(x)-\varphi_{2}(x)<\varphi_{1}(x)-\psi(x), \quad x \in\left(x_{0}, c\right) .
$$

Applying Lemma 1 for $f:=\varphi_{1}-\psi, g:=\varphi_{1}-\varphi_{2}$ and $k:=n-1$ we get $g^{n-1}\left(x_{0}\right)=0$, and consequently, $\varphi_{1}^{n-1}\left(x_{0}\right)=\varphi_{2}^{n-1}\left(x_{0}\right)$. Combining this with (6) we obtain

$$
\varphi_{1}^{i}\left(x_{0}\right)=\varphi_{2}^{i}\left(x_{0}\right), \quad i=0,1, \ldots, n-1 .
$$

Since $F$ is an $H_{n}$-family on $(a, b), \varphi_{1}(x)=\varphi_{2}(x)$ for $x \in(a, b)$, contrary to (7). This proves the lemma.

Lemma 5. Let $F$ and $\varphi$ be as in Lemma 4. If for every $k \in\{2, \ldots, n\}$ and for any positive integers $i_{1}, \ldots, i_{k}$ such that $i_{1}+\ldots+i_{k}=n, \varphi$ satisfies $\widetilde{W}_{n}\left(i_{1}, \ldots, i_{k} ; F\right)$ on $(a, b)$, then for every $k \in\{1, \ldots, n\}$ and for any positive integers $i_{1}, \ldots, i_{k}$ such that $i_{1}+\ldots+i_{k}=n, \varphi$ satisfies $W_{n}\left(i_{1}, \ldots, i_{k} ; F\right)$ on $(a, b)$.

Proof. It follows from Lemma 4 that $\varphi$ satisfies $W_{n}(n ; F)$ on $(a, b)$. Since $\varphi$ satisfies $\widetilde{W}_{n}\left(1^{(n)} ; F\right)$ on $(a, b)$, it also satisfies $W_{n}\left(1^{(n)} ; F\right)$ on $(a, b)$. This means that we need only consider $k \in\{2, \ldots, n-1\}$.

Fix $k \in\{2, \ldots, n-1\}$ and positive integers $i_{1}, \ldots, i_{k}$ such that $i_{1}+\ldots$ $+i_{k}=n$. We now prove that $\varphi$ satisfies $W_{n}\left(i_{1}, \ldots, i_{k} ; F\right)$ on $(a, b)$, i.e., for any $a<x_{1}<\ldots<x_{k}<b$,

$$
\operatorname{sgn}\left(\psi(x)-\varphi\left(x_{1}^{i_{1}}, \ldots, x_{k}^{i_{k}} ; \psi ; x\right)\right)=\operatorname{sgn}\left(\prod_{j=1}^{k}\left(x-x_{j}\right)^{i_{j}}\right)
$$

for $x \in(a, b)$. Since $\psi$ satisfies $\widetilde{W}_{n}\left(i_{1}, \ldots, i_{k} ; F\right)$ on $(a, b),(8)$ holds on $\left(x_{1}, x_{k}\right)$. We now show (8) holds on $\left(x_{k}, b\right)$; the proof for $\left(a, x_{1}\right)$ is analogous. It suffices to prove that

$$
\psi(x)>\varphi\left(x_{1}^{i_{1}}, \ldots, x_{k}^{i_{k}} ; \psi ; x\right), \quad x \in\left(x_{k}, b\right) .
$$

Set $\varphi_{1}(x):=\varphi\left(x_{1}^{i_{1}}, \ldots, x_{k}^{i_{k}} ; \psi ; x\right), x \in(a, b)$.

Assume that (9) does not hold and consider, as in the proof of Lemma 4, two cases:

1. $\psi(x) \geq \varphi_{1}(x)$ for $x \in\left(x_{k}, b\right)$ and $\psi(c)=\varphi_{1}(c)$ for a $c \in\left(x_{k}, b\right)$;

2. $\psi(c)<\varphi_{1}(c)$ for a $c \in\left(x_{k}, b\right)$.

1. Let $i_{k}=1$ and $\varphi_{2}(x):=\varphi\left(x_{1}^{i_{1}}, \ldots, x_{k-1}^{i_{k-1}}, c^{1} ; \psi ; x\right)$ for $x \in(a, b)$. Hence

$$
\psi(x)<\varphi_{2}(x), \quad x \in\left(x_{k-1}, c\right),
$$

because $\psi$ satisfies $\widetilde{W}_{n}\left(i_{1}, \ldots, i_{k-1}, 1 ; F\right)$ on $(a, b)$. By the definitions of $\varphi_{1}, \varphi_{2}$ and from the equality $\psi(c)=\varphi_{1}(c)$ we get 


$$
\begin{aligned}
\varphi_{1}^{j_{l}}\left(x_{l}\right) & =\varphi_{2}^{j_{l}}\left(x_{l}\right), \quad l=1, \ldots, k-1, j_{l}=0, \ldots, i_{l}-1, \\
\varphi_{1}(c) & =\varphi_{2}(c) .
\end{aligned}
$$

Therefore $\varphi_{1}(x)=\varphi_{2}(x)$ for $x \in(a, b)$. Combining this with (10) we obtain $\psi(x)<\varphi_{1}(x)$ for $x \in\left(x_{k}, c\right)$, contrary to 1 . If $i_{k}>1$, then considering the function $\varphi\left(x_{1}^{i_{1}}, \ldots, x_{k-1}^{i_{k-1}}, x_{k}^{i_{k}-1}, c^{1} ; \psi ; x\right)$ we get the same contradiction as for $i_{k}=1$.

2. Let $i_{k}=1$. Then there is a $p \in\{1, \ldots, k-1\}$ such that $i_{p}>1$. Set

$\varphi_{3}(x):=\varphi\left(x_{1}^{i_{1}}, \ldots, x_{p-1}^{i_{p-1}}, x_{p}^{i_{p}-1}, x_{p+1}^{i_{p+1}}, \ldots, x_{k}^{i_{k}}, c^{1} ; \psi ; x\right), \quad x \in(a, b)$.

Since $\psi$ satisfies $\widetilde{W}_{n}\left(i_{1}, \ldots, i_{k} ; F\right)$ and $\widetilde{W}_{n}\left(i_{1}, \ldots, i_{p-1}, i_{p}-1, i_{p+1}, \ldots\right.$ $\left.\ldots, i_{k}, 1 ; F\right)$ on $(a, b)$, it follows that

$$
\begin{array}{ll}
\psi(x)<\varphi_{1}(x), & x \in\left(x_{k-1}, x_{k}\right), \\
\psi(x)<\varphi_{3}(x), & x \in\left(x_{k}, c\right) .
\end{array}
$$

From the definitions of $\varphi_{1}$ and $\varphi_{3}$ and from the inequality $\psi(c)<\varphi_{1}(c)$, it may be concluded that

$$
\begin{array}{ll}
\varphi_{1}^{j_{l}}\left(x_{l}\right)=\varphi_{3}^{j_{l}}\left(x_{l}\right), \quad l \in\{1, \ldots, k\} \backslash\{p\}, j_{l}=0, \ldots, i_{l}-1, \\
\varphi_{1}^{j_{p}}\left(x_{p}\right)=\varphi_{3}^{j_{p}}\left(x_{p}\right), \quad j_{p}=0, \ldots, i_{p}-2, \\
\varphi_{1}(c)>\varphi_{3}(c) .
\end{array}
$$

We deduce from (13) and (14) that $\varphi_{1}(x) \neq \varphi_{3}(x)$ for $x \in(a, b) \backslash\left\{x_{1}, \ldots, x_{k}\right\}$; hence and from (14) we have $\varphi_{1}(x)>\varphi_{3}(x)$ for $x \in\left(x_{k}, b\right)$. Combining this with (12) we obtain

$$
\psi(x)<\varphi_{3}(x)<\varphi_{1}(x), \quad x \in\left(x_{k}, c\right) .
$$

It follows from (11), (15) and from the equality $\psi\left(x_{k}\right)=\varphi_{1}\left(x_{k}\right)$ that $\psi^{1}\left(x_{k}\right)=\varphi_{1}^{1}\left(x_{k}\right)$. We can rewrite $(15)$ as

$$
0<\varphi_{1}(x)-\varphi_{3}(x)<\varphi_{1}(x)-\psi(x), \quad x \in\left(x_{k}, c\right) .
$$

Applying Lemma 1 for $f:=\varphi_{1}-\psi, g:=\varphi_{1}-\varphi_{3}, k:=1$ and for $x_{0}:=x_{k}$ we get $g^{1}\left(x_{k}\right)=0$. Hence $\varphi_{1}^{1}\left(x_{k}\right)=\varphi_{3}^{1}\left(x_{k}\right)$. From this and from (13), we conclude that $\varphi_{1}(x)=\varphi_{3}(x)$ for $x \in(a, b)$, which is impossible by (14).

Let $i_{k}>1$. Put

$$
\varphi_{4}(x):=\varphi\left(x_{1}^{i_{1}}, \ldots, x_{k-1}^{i_{k-1}}, x_{k}^{i_{k}-1}, c^{1} ; \psi ; x\right), \quad x \in(a, b) .
$$

Analysis similar to that in the case where $i_{k}=1$ shows that

$$
\psi(x)<\varphi_{4}(x)<\varphi_{1}(x), \quad x \in\left(x_{k}, c\right)
$$

and $\varphi_{1}^{i_{k}-1}\left(x_{k}\right)=\varphi_{4}^{i_{k}-1}\left(x_{k}\right)$, by Lemma 1 . Hence, we have $\varphi_{1}(x)=\varphi_{2}(x)$ for $x \in(a, b)$, which gives the same contradiction as for $i_{k}=1$. This ends the proof. 
Lemma 6. Let $F$ and $\psi$ be as in Lemma 4. Assume that for every $j \in\{1, \ldots, n-1\}$ and for any points $a<x_{1}<\ldots<x_{k}<b(k:=n-j+1)$,

(16) $\operatorname{sgn}\left(\psi(x)-\varphi\left(x_{1}^{j}, x_{2}^{1}, \ldots, x_{k}^{1} ; \psi ; x\right)\right)$

$$
=\operatorname{sgn}\left(\left(x-x_{1}\right)^{j}\left(x-x_{2}\right) \ldots\left(x-x_{k}\right)\right)
$$

for $x \in\left(a, x_{k}\right)$. Then for every $i \in\{1, \ldots, n-1\}, \psi$ satisfies $W_{n}\left(i, 1^{(n-i)} ; F\right)$ on $(a, b)$.

The proof is similar to the proof of Lemma 5 for $i_{k}=1$, so we omit it.

Lemma 7. Let $F$ and $\psi$ be as in Lemma 4 . If $\psi$ is strictly $F$-convex on $(a, b)$, then for every $i \in\{1, \ldots, n\}, \psi$ satisfies $W_{n}\left(i, 1^{(n-i)} ; F\right)$ on $(a, b)$.

Proof. The proof is by induction on $n$. It follows from Lemma 4 (cf. [2]) that the lemma holds for $n=2$. Assume that it holds for $n-1(n \geq 3)$. Let $F$ be an $H_{n}$-family on $(a, b)$, let $\psi$ be $n-1$ times differentiable on $(a, b)$, and suppose that $\psi$ is strictly $F$-convex on $(a, b)$.

First we prove that $\psi$ satisfies $W_{n}\left(i, 1^{(n-i)} ; F\right)$ on $(a, b)$ for $i=1, \ldots$ $\ldots, n-1$. To do this, it suffices to show that the assumptions of Lemma 6 hold. If $j=1$, then $k=n$ and for every $a<x_{1}<\ldots<x_{n}<b$, (16) holds on $(a, b)$, because $\psi$ is strictly $F$-convex on $(a, b)$. Fix $j \in\{2, \ldots, n-1\}$ and $a<x_{1}<\ldots<x_{k}<b(k=n-j+1)$. We will prove (16) on $\left(a, x_{k}\right)$. Set

$$
G_{1}:=\left\{\left.\varphi\right|_{\left(a, x_{k}\right)}: \varphi \in F, \varphi\left(x_{k}\right)=\psi\left(x_{k}\right)\right\}, \quad \psi_{1}:=\left.\psi\right|_{\left(a, x_{k}\right)} .
$$

It is easy to check that $G_{1}$ is an $H_{n-1}$-family on $\left(a, x_{k}\right)$ and $\psi_{1}$ is strictly $G_{1}$-concave on $\left(a, x_{k}\right)$. Hence, from the inductive assumption and from Lemma 2 , we conclude that $\psi_{1}$ satisfies $K_{n-1}\left(j, 1^{(n-j-1)} ; G_{1}\right)$ on $\left(a, x_{k}\right)$. This implies

$$
\begin{aligned}
\operatorname{sgn}\left(\psi_{1}(x)\right. & -\bar{\varphi}(x)) \\
& =-\operatorname{sgn}\left(\left(x-x_{1}\right)^{j}\left(x-x_{2}\right) \ldots\left(x-x_{k-1}\right)\right), \quad x \in\left(a, x_{k}\right),
\end{aligned}
$$

where $\bar{\varphi} \in G_{1}$ is determined by the conditions

$$
\begin{aligned}
& \bar{\varphi}^{l}\left(x_{1}\right)=\psi_{1}^{l}\left(x_{1}\right), \quad l=0, \ldots, j-1, \\
& \bar{\varphi}\left(x_{p}\right)=\psi_{1}\left(x_{p}\right), \quad p=2, \ldots, k-1 .
\end{aligned}
$$

It follows from the definitions of $G_{1}, \psi_{1}$, and $\bar{\varphi}$ that

$$
\begin{aligned}
\bar{\varphi}(x) & =\varphi\left(x_{1}^{j}, x_{2}^{1}, \ldots, x_{k}^{1} ; \psi ; x\right), & & x \in\left(a, x_{k}\right), \\
\psi_{1}(x) & =\psi(x), & & x \in\left(a, x_{k}\right) .
\end{aligned}
$$

Therefore, we can rewrite (17) as

$$
\begin{aligned}
\operatorname{sgn}(\psi(x)- & \left.\varphi\left(x_{1}^{j}, x_{2}^{1}, \ldots, x_{k}^{1} ; \psi ; x\right)\right) \\
& =-\operatorname{sgn}\left(\left(x-x_{1}\right)^{j}\left(x-x_{2}\right) \ldots\left(x-x_{k-1}\right)\right), \quad x \in\left(a, x_{k}\right) .
\end{aligned}
$$

Combining this with $x-x_{k}<0$ for $x \in\left(a, x_{k}\right)$ we get (16) on $\left(a, x_{k}\right)$. 
The proof will be completed as soon as we can show that $\psi$ satisfies $W_{n}(n ; F)$ on $(a, b)$. To do this, it is sufficient, by Lemma 4 (we have already proved that $\psi$ satisfies $W_{n}(n-1,1 ; F)$ on $\left.(a, b)\right)$, to prove that $\psi$ satisfies $\widetilde{W}_{n}(1, n-1 ; F)$ on $(a, b)$. Let $a<x_{1}<x_{2}<b$. We have to show that

$$
\begin{aligned}
\operatorname{sgn}\left(\psi(x)-\varphi\left(x_{1}^{1}, x_{2}^{n-1} ; \psi ; x\right)\right) & \\
& =\operatorname{sgn}\left(\left(x-x_{1}\right)\left(x-x_{2}\right)^{n-1}\right), \quad x \in\left(x_{1}, x_{2}\right) .
\end{aligned}
$$

Define

$$
G_{2}:=\left\{\left.\varphi\right|_{\left(x_{1}, b\right)}: \varphi \in F, \varphi\left(x_{1}\right)=\psi\left(x_{1}\right)\right\}, \quad \psi_{2}:=\left.\psi\right|_{\left(x_{1}, b\right)} .
$$

Obviously, $G_{2}$ is an $H_{n-1}$-family on $\left(x_{1}, b\right)$ and $\psi_{2}$ is strictly $G_{2}$-convex on $\left(x_{1}, b\right)$. Hence, from the inductive assumption we deduce that $\psi_{2}$ satisfies $W_{n-1}\left(n-1 ; G_{2}\right)$ on $\left(x_{1}, b\right)$. An analysis similar to that used in the first part of the proof shows that

$$
\operatorname{sgn}\left(\psi(x)-\varphi\left(x_{1}^{1}, x_{2}^{n-1} ; \psi ; x\right)\right)=\operatorname{sgn}\left(\left(x-x_{2}\right)^{n-1}\right), \quad x \in\left(x_{1}, b\right) .
$$

Combining this with $x-x_{1}>0$ for $x \in\left(x_{1}, b\right)$ we get (18), which completes the proof of the lemma.

3. Main results. In this section we give necessary and sufficient conditions for strict convexity with the use of the conditions $W_{n}\left(i_{1}, \ldots, i_{k} ; F\right)$. First we prove that if $\psi$ is strictly $F$-convex, then $\psi$ satisfies every condition $W_{n}\left(i_{1}, \ldots, i_{k} ; F\right)$.

THEOREM 1. Let $F$ be an $H_{n}$-family on $(a, b)$ and let $\psi$ be $n-1$ times differentiable on $(a, b)$. If $\psi$ is strictly $F$-convex on $(a, b)$, then for any positive integers $i_{1}, \ldots, i_{k}$ such that $i_{1}+\ldots+i_{k}=n, \psi$ satisfies $W_{n}\left(i_{1}, \ldots, i_{k} ; F\right)$ on $(a, b)$.

Proof. The proof is by induction on $n$. It follows from Lemma 4 (cf. [2]) that the statement holds for $n=2$. Assume it holds for $2, \ldots, n-1(n \geq 3)$.

Let $F$ and $\psi$ be as in the statement of the theorem. By Lemma 5, it suffices to show that for every $k \in\{2, \ldots, n\}$ and for any positive integers $i_{1}, \ldots, i_{k}$ such that $i_{1}+\ldots+i_{k}=n, \psi$ satisfies $\widetilde{W}_{n}\left(i_{1}, \ldots, i_{k} ; F\right)$ on $(a, b)$. Since $k \geq 2, i_{1} \leq n-1$. If $i_{1}=n-1$, then $k=2$ and $i_{2}=1$. By Lemma 7 , $\psi$ satisfies $W_{n}(n-1,1 ; F)$ on $(a, b)$. Therefore we need only consider the case $i_{1} \leq n-2$.

Fix $k \in\{2, \ldots, n\}$, positive integers $i_{1}, \ldots, i_{k}$ such that $i_{1}+\ldots+i_{k}=n$ and $i_{1} \leq n-2$, and points $a<x_{1}<\ldots<x_{k}<b$. If we prove that

$$
\operatorname{sgn}\left(\psi(x)-\varphi\left(x_{1}^{i_{1}}, \ldots, x_{k}^{i_{k}} ; \psi ; x\right)\right)=\operatorname{sgn}\left(\prod_{j=1}^{k}\left(x-x_{j}\right)^{i_{j}}\right), \quad x \in\left(x_{1}, x_{k}\right),
$$


the assertion follows. Put

$$
\begin{aligned}
G_{1} & :=\left\{\left.\varphi\right|_{\left(x_{1}, b\right)}: \varphi \in F, \varphi^{j}\left(x_{1}\right)=\psi^{j}\left(x_{1}\right), j=0, \ldots, i_{1}-1\right\}, \\
\psi_{1} & :=\left.\psi\right|_{\left(x_{1}, b\right)} .
\end{aligned}
$$

It is easily seen that $G_{1}$ is an $H_{n-i_{1}}$-family on $\left(x_{1}, b\right)$. By Lemma 7 , $\psi$ satisfies $W_{n}\left(i_{1}, 1^{\left(n-i_{1}\right)} ; F\right)$ on $(a, b)$. Consequently, $\psi_{1}$ is strictly $G_{1}$-convex on $\left(x_{1}, b\right)$. Hence and from the inductive assumption we see that $\psi_{1}$ satisfies $W_{n-i_{1}}\left(i_{2}, \ldots, i_{k} ; G_{1}\right)$ on $\left(x_{1}, b\right)$. This implies that

$$
\operatorname{sgn}\left(\psi_{1}(x)-\varphi(x)\right)=\operatorname{sgn}\left(\prod_{j=2}^{k}\left(x-x_{j}\right)^{i_{j}}\right), \quad x \in\left(x_{1}, b\right),
$$

where $\varphi \in G_{1}$ is determined by the conditions

$$
\varphi^{j_{l}}\left(x_{l}\right)=\psi^{j_{l}}\left(x_{l}\right), \quad l=2, \ldots, k, j_{l}=0, \ldots, i_{l}-1 .
$$

It follows from the definitions of $G_{1}, \varphi$ and $\psi_{1}$ that

$$
\begin{aligned}
\varphi(x) & =\varphi\left(x_{1}^{i_{1}}, \ldots, x_{k}^{i_{k}} ; \psi ; x\right), & & x \in\left(x_{1}, b\right), \\
\psi_{1}(x) & =\psi(x), & & x \in\left(x_{1}, b\right) .
\end{aligned}
$$

Therefore, we can rewrite (20) as

$$
\operatorname{sgn}\left(\psi(x)-\varphi\left(x_{1}^{i_{1}}, \ldots, x_{k}^{i_{k}} ; \psi ; x\right)\right)=\operatorname{sgn}\left(\prod_{j=2}^{k}\left(x-x_{j}\right)^{i_{j}}\right), \quad x \in\left(x_{1}, b\right) .
$$

Since $\left(x-x_{1}\right)^{i_{1}}>0$ for $x \in\left(x_{1}, b\right)$, we get (19), which completes the proof.

Now we will be concerned with sufficient conditions for strict convexity.

TheOREM 2. Let $F$ and $\psi$ be as in Theorem 1 . If $\psi$ satisfies $W_{n}\left(i_{1}, \ldots\right.$ $\left.\ldots, i_{k} ; F\right)$ on $(a, b)$ for some $i_{1}, \ldots, i_{k} \in\{1,2,3\}$ such that $i_{1}+\ldots+i_{k}=n$, then $\psi$ is strictly $F$-convex on $(a, b)$.

To prove this theorem we need the following

LEMMA 8. Let $G$ be an $H_{r}$-family on $(c, d)(r \geq 4)$ and let $\psi$ be $r-1$ times differentiable on $(c, d)$. If $\psi$ satisfies $W_{r}\left(i_{1}, \ldots, i_{k} ; G\right)$ on $(c, d)$, where $i_{1}, \ldots, i_{k} \in\{1,2,3\}, i_{1}+\ldots+i_{k}=r, i_{k} \neq 1$ and Theorem 2 holds for $n=n_{1}:=i_{2}+\ldots+i_{k}$, then $\psi$ satisfies $W_{r}\left(i_{1}, \ldots, i_{k-1}, i_{k}-1,1 ; G\right)$ on $(c, d)$.

Proof. Fix $c<x_{1}<\ldots<x_{k}<x_{k+1}<d$ and set

$$
\varphi_{1}(x):=\varphi\left(x_{1}^{i_{1}}, \ldots, x_{k-1}^{i_{k-1}}, x_{k}^{i_{k}-1}, x_{k+1}^{1} ; \psi ; x\right), \quad x \in(c, d) .
$$

If we prove that

$$
\operatorname{sgn}\left(\psi(x)-\varphi_{1}(x)\right)=\operatorname{sgn}\left(\left(x-x_{k}\right)^{i_{k}-1}\left(x-x_{k+1}\right) \prod_{l=1}^{k-1}\left(x-x_{l}\right)^{i_{l}}\right)
$$


for $x \in(c, d)$, the assertion follows. Put

$$
\begin{aligned}
F & :=\left\{\left.\varphi\right|_{\left(x_{1}, d\right)}: \varphi \in G, \varphi^{j}\left(x_{1}\right)=\psi^{j}\left(x_{1}\right), j=0, \ldots, i_{1}-1\right\}, \\
\psi_{1} & :=\left.\psi\right|_{\left(x_{1}, d\right)} .
\end{aligned}
$$

Obviously, $F$ is an $H_{n_{1}}$-family on $\left(x_{1}, d\right)$. The function $\psi_{1}$ satisfies $W_{n_{1}}\left(i_{2}, \ldots\right.$ $\left.\ldots, i_{k} ; F\right)$ on $\left(x_{1}, d\right)$, because $\psi$ satisfies $W_{r}\left(i_{1}, \ldots, i_{k} ; G\right)$ on $(c, d)$. Since Theorem 2 was assumed to hold for $n=n_{1}, \psi_{1}$ is strictly $F$-convex on $\left(x_{1}, d\right)$. By Theorem $1, \psi$ satisfies $W_{n_{1}}\left(i_{2}, \ldots, i_{k}-1,1 ; F\right)$ on $\left(x_{1}, d\right)$. It follows that

$$
\begin{aligned}
& \operatorname{sgn}\left(\psi_{1}(x)-\bar{\varphi}(x)\right) \\
& \quad=\operatorname{sgn}\left(\left(x-x_{k}\right)^{i_{k}-1}\left(x-x_{k+1}\right) \prod_{l=2}^{k-1}\left(x-x_{l}\right)^{i_{l}}\right), \quad x \in\left(x_{1}, d\right),
\end{aligned}
$$

where $\bar{\varphi} \in F$ is determined by the conditions

$$
\begin{array}{rlrl}
\bar{\varphi}^{j_{l}}\left(x_{l}\right) & =\psi_{1}^{j_{l}}\left(x_{l}\right), \quad l=2, \ldots, k-1, j_{l}=0, \ldots, i_{l}-1, \\
\bar{\varphi}^{j_{k}}\left(x_{k}\right) & =\psi_{1}^{j_{k}}\left(x_{k}\right), \quad j_{k}=0, \ldots, i_{k}-2, \\
\bar{\varphi}\left(x_{k+1}\right) & =\psi_{1}\left(x_{k+1}\right) . & &
\end{array}
$$

By the definition of $\bar{\varphi}, \psi_{1}$ and $F$ we have

$$
\bar{\varphi}(x)=\varphi_{1}(x), \quad \psi_{1}(x)=\psi(x), \quad x \in\left(x_{1}, d\right) .
$$

Combining these with (22) we get $(21)$ on $\left(x_{1}, d\right)$, because $\left(x-x_{1}\right)^{i_{1}}>0$ for $x \in\left(x_{1}, d\right)$. We only have to show that (21) holds on $\left(c, x_{1}\right)$. To do this, consider

$$
\varphi_{2}(x):=\varphi\left(x_{1}^{i_{1}}, \ldots, x_{k-1}^{i_{k-1}}, x_{k}^{i_{k}} ; \psi ; x\right), \quad x \in(c, d) .
$$

Since $\psi$ satisfies $W_{r}\left(i_{1}, \ldots, i_{k} ; G\right)$ on $(c, d)$,

$$
\operatorname{sgn}\left(\psi(x)-\varphi_{2}(x)\right)=\operatorname{sgn}\left(\prod_{l=1}^{k}\left(x-x_{l}\right)^{i_{l}}\right), \quad x \in(c, d) .
$$

Hence

$$
(-1)^{r} \varphi_{2}(x)<(-1)^{r} \psi(x), \quad x \in\left(c, x_{1}\right) .
$$

By the definition of $\varphi_{1}$ we have $\varphi_{1}\left(x_{k+1}\right)=\psi\left(x_{k+1}\right)$, and $\psi\left(x_{k+1}\right)>$ $\varphi_{2}\left(x_{k+1}\right)$ from (23). Therefore

$$
\varphi_{1}\left(x_{k+1}\right)>\varphi_{2}\left(x_{k+1}\right) .
$$

From the definitions of $\varphi_{1}$ and $\varphi_{2}$ we get

$$
\begin{aligned}
\varphi_{1}^{j_{l}}\left(x_{l}\right) & =\varphi_{2}^{j_{l}}\left(x_{l}\right), \quad l=1, \ldots, k-1, j_{l}=0, \ldots, i_{l}-1, \\
\varphi_{1}^{j_{k}}\left(x_{k}\right) & =\varphi_{2}^{j_{k}}\left(x_{k}\right), \quad j_{k}=0, \ldots, i_{k}-2,
\end{aligned}
$$


and $i_{1}+\ldots+i_{k-1}+\left(i_{k}-1\right)=r-1$. Hence, from the definition of an $H_{r}$-family $\left(\varphi_{1}, \varphi_{2} \in G\right)$ and from $(25)$ we obtain

$$
(-1)^{r-1} \varphi_{1}(x)>(-1)^{r-1} \varphi_{2}(x), \quad x \in\left(c, x_{1}\right),
$$

which gives

$$
(-1)^{r} \varphi_{1}(x)<(-1)^{r} \varphi_{2}(x), \quad x \in\left(c, x_{1}\right) .
$$

Combining this with $(24)$ we see that

$$
(-1)^{r} \varphi_{1}(x)<(-1)^{r} \psi(x), \quad x \in\left(c, x_{1}\right),
$$

which implies $(21)$ on $\left(c, x_{1}\right)$ and the proof is complete.

Proof of Theorem 2. For $n=2$ and $n=3$ the theorem is true. Assume that it holds for $2,3, \ldots, n-1(n \geq 4)$.

Let $F$ and $\psi$ be as in the statement of the theorem. By Lemma 8, it suffices to consider the case $i_{k}=1$.

If we prove that $\psi$ satisfies $\widetilde{W}_{n}\left(1^{(n)} ; F\right)$ on $(a, b)$, the assertion follows. Fix $a<x_{1}<\ldots<x_{n}<b$ and let $\varphi_{1}(x):=\varphi\left(x_{1}^{1}, \ldots, x_{n}^{1} ; \psi ; x\right)$ for $x \in(a, b)$. We have to show that

$$
\operatorname{sgn}\left(\psi(x)-\varphi_{1}(x)\right)=\operatorname{sgn}\left(\prod_{l=1}^{n}\left(x-x_{l}\right)\right), \quad x \in\left(x_{1}, x_{n}\right) .
$$

Set

$$
G:=\left\{\left.\varphi\right|_{\left(a, x_{n}\right)}: \varphi \in F, \varphi\left(x_{n}\right)=\psi\left(x_{n}\right)\right\}, \quad \psi_{1}:=\left.\psi\right|_{\left(a, x_{n}\right)} .
$$

Obviously, $G$ is an $H_{n-1}$-family on $\left(a, x_{n}\right)$. The function $\psi_{1}$ satisfies $K_{n-1}\left(i_{1}, \ldots, i_{k-1} ; G\right)$ on $\left(a, x_{n}\right)$, because $\psi$ satisfies $W_{n}\left(i_{1}, \ldots, i_{k} ; F\right)$ on $(a, b)$ and $i_{k}=1$. Hence, from the inductive assumption and from Lemma 3 we conclude that $\psi_{1}$ is strictly $G$-concave on $\left(a, x_{n}\right)$. This implies that

$$
\operatorname{sgn}\left(\psi(x)-\varphi_{1}(x)\right)=-\operatorname{sgn}\left(\prod_{l=1}^{n-1}\left(x-x_{l}\right)\right), \quad x \in\left(a, x_{n}\right) .
$$

Since $x-x_{n}<0$ for $x \in\left(a, x_{n}\right),(27)$ gives (26) and the proof is complete.

One may ask whether Theorem 2 is true if some $i_{j}>3$. We have not been able to settle this question.

Theorems 1 and 2 may be summarized as follows:

TheOREM 3. Let $F$ and $\psi$ be as in Theorem 1 . If $\psi$ satisfies $W_{n}\left(i_{1}, \ldots\right.$ $\left.\ldots, i_{k} ; F\right)$ on $(a, b)$ for some $i_{1}, \ldots, i_{k} \in\{1,2,3\}$ such that $i_{1}+\ldots+i_{k}=n$, then $\psi$ satisfies $W_{n}\left(i_{1}, \ldots, i_{k} ; F\right)$ on $(a, b)$ for any positive integers $i_{1}, \ldots, i_{k}$ such that $i_{1}+\ldots+i_{k}=n$.

Similar results can be obtained for strict concavity.

Using an analogous reasoning one can get similar results for convexity and concavity. 


\section{References}

[1] E. F. Beckenbach, Generalized convex functions, Bull. Amer. Math. Soc. 43 (1937), 363-371.

2] D. Brydak, Application of generalized convex functions to second order differential inequalities, in: General Inequalities 4 (4th International Conference on General Inequalities, Oberwolfach, 1983), W. Walter (ed.), Birkhäuser, 1984, 297-305.

[3] P. Hartman, Unrestricted n-parameter families, Rend. Circ. Mat. Palermo (2) 7 (1958), 123-142, MR 21\#4211.

[4] J. Krzyszkowski, On the generalized convex functions with respect to the threeparameters family of functions, Rocznik Naukowo-Dydaktyczny WSP w Krakowie, Prace Matematyczne XII, 115 (1987), 47-61.

[5] L. Tornheim, On n-parameter families of functions and associated convex functions, Trans. Amer. Math. Soc. 69 (1950), 457-467.

INSTITUTE OF MATHEMATICS

PEDAGOGICAL UNIVERSITY

PODCHORĄ̇̇YCH 2

30-084 KRAKÓW, POLAND

Reçu par la Rédaction le 2.12.1993

Révisé le 15.5.1994 et 8.12.1994 